\title{
Does pancreas transplantation influence the course of diabetic retinopathy?
}

\author{
A. Königsrainer, K. Miller, W. Steurer, G. Kieselbach, C. Aichberger, D. Öfner and R. Margreiter \\ Department of Transplant Surgery, University Hospital, Innsbruck, Austria
}

Summary. Between March 1983 and December 1989 a total of 57 pancreas transplants were performed in 54 patients, of whom 49 also received a kidney for end-stage diabetic nephropathy. Of the surviving 44 patients, 39 had regular pre-operative and post-operative ophthalmological examinations. Diabetic retinopathy was classified according to the original "Early treatment diabetic retinopathy study" (ETDRS) protocol. At the time of this analysis a total of 25 patients had a functioning pancreas transplant and 23 of them also a functioning renal allograft after a mean observation time of 43.2 months (Group 1). They were all free of exogenous insulin, HbAlc being $6.2 \%$ (5.1-6.9\%; normal value $4.2-5.9 \%$ ). Fourteen patients in Group 2 lost their pancreas transplant during the first four years. Six of them still have a functioning renal allograft, four patients regularly undergo hemodialysis. Mean $\mathrm{HbAlc}$ is $7.5 \%$ (5.7-9.2\%). Before transplantation, grade of retinopathy according the ETDRS protocol was $6.7(2-10)$ in group 1 patients and $7.9(3-10)$ in group 2. In group 1 patients stabilisation of retinopathy was observed in 33 eyes (73.3\%) and clear improvement achieved in 4 eyes (8.8\%). Detoriation occurred in 8 eyes $(17.7 \%)$ only. In group 2 , 14 eyes $(54 \%)$ remained stable, whereas progression of the disease continued in 12 eyes (46\%). From these results it is concluded that the course of diabetic retinopathy is positively influenced by successful pancreas transplantation.

Key words: Pancreatic transplantation - DiabetesRetinopathy

\section{Introduction}

Between 10 and 20 years following the onset of Type 1 (insulin-dependent) diabetes mellitus $50 \%$ of patients develop diabetic retinopathy, which if untreated leads to blindness within two years in a high percentage of patients. The risk of becoming blind can be significantly reduced by early laser therapy (Kampik \& Ulbig 1986). Furthermore, optimal control of carbohydrate metabolism and blood pressure are believed to be essential for a benign course of retinopathy. New techniques for treatment of diabetes such as intensified insulin therapy or continuous insulin injection by means of pumps are capable of controlling carbohydrate metabolism in a nearphysiological manner (Puklin et al.1982). A beneficial effect, however, to the course of secondary complications of diabetes has not yet been proven (Siperstein 1983). Normalisation of carbohydrate metabolism can currently be achieved only with a vascularised pancreas transplant (Lacy 1982). The question as to whether a successful pancreas transplantation provides this beneficial effect has not been sufficiently answered on the basis of clinical experience comprising over 3000 such patients (Ramsay et al. 1988; Kennedy et al. 1990; Solders et al. 1987). With respect to the course of retinopathy, our patients with a functioning pancreas transplant for more than 12 months were compared with those who lost the function of their graft.

\section{Patients and methods}

Between March 1983 and December 1989 a total of 57 pancreas transplants were performed in 54 Type 1 diabetic patients. In 49 instances this procedure was combined with a renal transplant for the treatment of end-stage diabetic nephropathy. Surgical techniques, immunosuppression and post-operative management are described in a previous section of this supplement (Königstainer et al). As of December $1990,44(82 \%)$ of the patients are still alive after a mean observation time of $47.2(13-92)$ months. Of these 44 patients, 26 $(61.3 \%)$ have a nomally-functioning pancreas graft and $31(70.4 \%)$ a well-functioning renal transplant. In a prospective study 39 of these patients $(88.8 \%)$ had a complete ophthalmological examination prior to transplantation and at four to six month intervals thereafter. The study protocol included clinical examination, determination of visual acuity, sterioscopic examination of the fundus and colour photography documentation. Retinopathy was graded by one examiner only according to the original early treatment diabetic retinopathy study system (ETDRS report number 2 1987). A two-grade change in either direction was classified as improvement or deterioration. For the long-term follow- 
up of pancreas transplants, urinary amylase was regularly measured. Endocrine monitoring included two daily blood glucose determinations carried out by the patients themselves; determination of the HbAlc once a month as well as oral glucose tolerance tests and C-peptide measurements four times per year. In order to study the effect of normalisation of carbohydrate metabolism on the course of diabetic retinopathy, 25 patients with a functioning graft (Group 1) were compared with 14 patients who lost the function of their graft between 1 day and 4 years following transplantation due either to technical failure or immunological reasons (Group 2).

Patient characteristics as well as their pre-operative ophthalmological status are depicted in Table 1 Three patients in Group 1 and 2 patients in Group 2 were blind in one eye One patient in Group 1 was already completely amaurotic at the time of transplantation. Altogether, 20 patients in Group 1 and 10 patients in the control group had panretinal laser coagulation pior to transplantation. A total of 45 eyes in Group 1 and 26 eyes in Group 2 were analysed.

Informed consent was obtained from all patients.

Table 1. Characteristics of Type1 (insulin-dependent) diabetic patient groups and ophthalmological status before pancreatic transplantation

\begin{tabular}{lll}
\hline & GROUP 1 & GROUP 2 \\
\hline Patients (n) & 25 & 14 \\
Age (years) & $38.0(27-59)$ & $32.8(19-51)$ \\
Sex (m /f) & $15 / 10$ & $6 / 8$ \\
Diabetes duration (years) & $23.6(13-37)$ & $20.0(12-29)$ \\
Time on dialysis & $28.6(0-156)$ & $20.8(0-70)$ \\
(months) & & \\
Eyes (n) & 45 & 26 \\
Blindness (cyes) & 5 & 2 \\
Grade of diabetic & $6.7(2-10)$ & $6.9(3-10)$ \\
retinopathy & \\
Photocoagulation (eyes) & 32 & 19 \\
\hline
\end{tabular}

\section{Results}

\section{Group I}

Of the 25 patients in Group 1,23 had both a functioning pancreatic and renal transplant. One female patient in this group received a second renal transplant after having lost the first one from chronic rejection.

Stabilisation of retinopathic lesions was observed in 33 eyes $(73.3 \%$ ) and deterioration in $8(17.7 \%$ ) (Table 2). A clear regression was demonstrable in both eyes of two patients who were transplanted with pre-proliferative stage of retinopathy. In one of these two patients who had grade 3 to 4 retinopathy prior to transplantation, no signs of retinopathy were detectable three years after grafting. All eyes with proliferative retinopathy at the time of transplantation benefitted from pancreas transplantation. Progression of the disease was halted or retarded in the majority of cases but not in every patient. Rapid progression following transplantation, however, did not occur in any patient (Table 3).
Table 2. Results and metabolic control during the observation time

\begin{tabular}{lll}
\hline & GROUP 1 & GROUP2 \\
\hline SKP & 24 & 10 \\
Functioning renal grafts (n) & 23 & 6 \\
Haemodialysis & 1 & 4 \\
Observation time (months) & $43.2(13-92)$ & $51.3(42-71)$ \\
HbA1c (no.value 4.2 & $6.2(5.1-6.9)$ & $7.5(5.7-9.2)$ \\
$5.9 \%)$ & \\
\hline
\end{tabular}

SKP: simultaneous kidney-pancreas transplantation

\section{Group 2:}

Six patients in Group 2 still have a functioning renal graft, four enjoy sufficient function of their own kidneys and four patients are on haemodialysis (Table 2). Stabilisation of retinopathy was observed in 14 eyes $(54 \%)$ and clear progression in a further 12 eyes (46\%). (Table 3)

Table 3. Course of diabetic retinopathy after pancreatic transplantation

\begin{tabular}{lll}
\hline & GROUP 1 & GROUP 2 \\
\hline Regression & $4(8.8 \%)$ & \\
Stabilization & $33(73.3 \%)$ & $14(54 \%)$ \\
Progression & $8(17.7 \%)$ & $12(46 \%)$ \\
\hline
\end{tabular}

Chi - square $7.967 \quad p<0.03$

\section{Discussion}

The long-term goal of pancreas transplantation must be control of blood glucose but also the prevention of secondary complications. In order to prove this, pancreas transplantation must be carried out at a very early stage of the disease. The poor results achieved so far with single pancreas transplantation together with all the side effects of life-long pharmacological immunosuppression do not currently justify replacement of exogenous insulin therapy by pancreas transplantation (Pyke 1990) Furthermore, it is very difficult at that stage to predict who is going to develop secondary complications of diabetes. Currently, pancreas transplantation is performed by most groups in patients suffering from end-stage diabetic nephropathy. By this time, not only the kidneys but also other organs and tissues have already been severely damaged. Somewhat in contrast to the available literature on this subject (Lacy 1982), we believe that our findings do indeed provide some evidence that a wellfunctioning pancreas transplant has a beneficial effect on the course of diabetic retinopathy (Königsrainer et al.1988). Since the number of patients in our study is small and together with the fact that the patient population in Group 2 is rather inhomogenous regarding renal function, no firm conclusion can be drawn from this study. No improvement of the disease has been observed in patients with an adequately functioning renal transplant only. The percentage of stabilisation is much higher and the percentage of progression much lower among patients with a pancreas 
graft, when compared to Group 2 patients. Since only 4 patients in Group 2 require haemodialysis, improvement or stabilisation in Group 1 patients cannot be ascribed to correction of uraemia only. The 2 patients with a clear improvement in both eyes provide some evidence that a beneficial effect can be expected when pancreas transplantation is performed at a very early stage of retinopathy. These observations may be taken as a hint that timely pancreas transplantation is capable of preventing diabetic retinopathy.

\section{References}

Early treatment diabetic retinopathy study report number 2 (1987) Treatment techniques and clinical guidelines for photocoagulation of diabetic macular edema. Ophthalmology 94: $761-774$

Kampik A, Ulbig M (1986) Is proliverative diabetic retinopathy an indication

for pancreatic transplantation? Transplant Proc 18: 62-63

Kennedy WR, Navarro X, Goetz FC, Sutherland DER, Najarian IS (1990) Effects of pancreatic transplantation on diabetic neuropathy. N Engl J Med 322: 1031-1037
Königsrainer A, Miller K, Keiselbach G, Margreiter R (1988) Progression of diabetic retionpathy after pancreas transplantation. N Engl J Med 319: 247

Lacy PE (1982) Pancreatic transplantation as a means of insulin delivery . Diabetes Care 5: 93-97

Puklin JE, Tamborlone WV, Felig P, Genel M, Sherwin RS (1982) Influence of long-term insulin infusion pump treatment of Type I diabetic on diabetic retinopathy. Ophthomolgy 89: 735-747

Pyke DA (1990) A Critque of pancreas transplantation. Clin Transplantation 4: $235-237$

Ramsay RC, Goetz FC, Sutherland DER, Mauer SM, Robison LL, Cantrill HL, Knobloch WH, Najarian JS (1988) Progression of diabetic retinopathy after pancreas transplantation for insulindependent diabetes mellitus. $\mathrm{N}$ Bngl J Med 318: 208-214

Siperstein MD (1983) Diabetic microangiopathy and the control of blood glucose. N Engl J Med 309: 1577-1579

Solders $G$, Gunnarsson R, Persson A, Wilczek H, Tyden G, Groth CG (1987) Effects of combined pancreatic and renal transplantation on diabetic neuropathy: a two-year follow-up study, Lancet, November 28: 1232-1235

Dr. Alfred Königsrainer

Dep. Transplant Surgery - University Hospital

Anichstrasse 35

A-6020IN N S B R U C K 